\title{
Left invertibility of output-quantized MISO linear systems
}

\author{
Nevio Dubbini * Maurizio Monge ${ }^{* *}$ Antonio Bicchi ${ }^{*}$ \\ *Interdepartmental research center “E. Piaggio”, University of Pisa, Italy \\ (e-mail: nevio.dubbini@for.unipi.it) \\ ** Scuola Normale Superiore, Pisa, Italy
}

\begin{abstract}
This paper studies left invertibility of single-output discrete-time quantized linear systems. Quantized outputs are generated according to a given partition of the state-space, while inputs are sequences on a finite alphabet. Left invertibility deals with the possibility of recovering unknown inputs from the only knowledge of the outputs. It is reduced, under suitable conditions, to left D-invertibility: while left invertibility takes into account membership to sets of a given partition, left D-invertibility considers only membership to a single set, and is easily (and algorithmically) detectable. Our main result is a sufficient condition for the equivalence between left invertibility and left D-invertibility in MISO system. In unidimensional systems the equivalence is valid except at most a finite (and computable) number of cases. These results allows the effective detection of left invertibility by means of left Dinvertibility, which is algorithmically detectable. An example with effective computations is presented to show the application of the proposed method.
\end{abstract}

Keywords: Left invertibility, uniform quantization, finite inputs, Kronecker's theorem, discrete time

\section{INTRODUCTION}

Left invertibility is an important problem of systems theory and deals with the possibility of recovering unknown inputs applied to the system from the knowledge of the outputs. We investigate left invertibility of discrete-time I/O quantized Multi-Input Single-Output (MISO) linear systems. In particular, inputs are arbitrary sequences of symbols in a finite alphabet: each symbol is associated to an action on the system. Information available on the system is represented by sequences of output values, generated by the system evolution according to a given partition of the state-space (uniform quantization here).

The mathematical operation of quantization has practical motivations in the following two classes of problems: the control with discrete sensors and/or actuators, which have finite resolution, and the control under communication constraints, like channels with limited capacity. Quantization has been studied since the late fifties (see for instance Bertram [1958], Kalman [1956], Szanier [1994]). Sampled-data systems and signal processing have been the first field of interest for quantization (Curry [1970]), while the idea of quantization as a nonlinearity pervades recent contributions on the subject (Delchamps [1990], Fu [2005]). The definition of a dynamic quantization scheme (Tatikonda [2004], Brockett [2000]) has been one of the most significant methodology introduced in the recent literature. In recent years there has been also a considerable amount of work on quantized control systems stimulated by the growing number of applications involving "networked" control systems,

\footnotetext{
* This work has been partially supported by the European Commission under contract IST 224428 (2008) "CHAT - Control of Heterogeneous Automation systems: Technologies for scalability, reconfigurability and security", and contributes to the goals of HYCON2, the Highly-complex and Networked Control Systems Network of Excellence, funded by the European Commission under FP7 with contract number FP7-ICT-2009-5-224053.
}

interconnected through channels of limited capacity (see e.g. Bicchi [1992], Carli [2008], Nair [2007], Tatikonda [2004]).

As to left invertibility, literature shows many works on both invertibility of linear (Brockett [1965], Silverman [1969]) and nonlinear systems. In Hirschorn [1979], Hirschorn2 [1979], Respondek [1990] invertibility of nonlinear continuous-time systems and in Fliess [1986] the notion of differential algebraic invertibility are discussed. A good compendium of left and right invertibility of both linear and nonlinear systems can be found in Respondek [2001]. In Tanwani [2008], Vu [2006] the left invertibility problem for switched systems is discussed. Applications of left invertibility include fault detection in Supervisory Control and Data Acquisition (SCADA) systems, system identification, and cryptography. The applicability of system inversion to fault detection in linear time-invariant systems was first demonstrated in Szigeti [2001]. In Edelmayer [2004] it is shown how fault detection of both linear and nonlinear systems can be viewed as an input reconstruction process. Moreover, dynamic inversion methods were successfully applied to many interesting problems in aerospace and aviation (Goodwin [2002], Krupadanam [2002]). As to applications to cryptography, it deals with the general construction of cryptosystems based on chaos (see Dubbini [2010] and reference therein).

The intent of this paper is to show that the analysis of left invertibility can be substituted, under suitable conditions, by the analysis of a stronger notion, left D-invertibility. While left invertibility takes in account whether two states are in the same element of a given partition, left D-invertibility considers only the membership to a single set. For this reason left Dinvertibility is easy to check, and it is algorithmically detectable (Theorem 1). The main results of this paper state condition under which left invertibility and left D-invertibility of MISO systems are equivalent. In particular, in unidimensional systems the two notions are equivalent except at most a finite 
number of cases (Theorem 5); in a MISO system uniform left D-invertibility and uniform left invertibility are (exactly) equivalent when the dynamic matrix has at least a trascendental eigenvalue (Corollary 1). The main mathematical tool used is a generalization of a (number theoretic) theorem of Kronecker, which has to do with density in the unit cube of the fractional parts of real numbers. By means of a particular construction the problem of "turning" left D-invertibility into left invertibility can be handled with a Kronecker-type density theorem.

The paper is organized as follows: section 2 contains the formalization of the problem under study, and some preliminary results. Section 3 shows the procedure to prove the equivalence between left D-invertibility and left invertibility and states the equivalence results for unidimensional systems. Section 4 concerns MISO system, applying results obtained in section 3 to prove equivalence results for this class of systems. In section 5 explicit calculations are done in a comprehensive example. Conclusions and future work are explained in section 6 .

\section{LEFT INVERTIBILITY AND FIRST RESULTS}

Notations: We indicate with $e_{i}$ the $i$-th vector of the canonical basis of $\mathbb{R}^{d}$; with $\left\langle v_{1}, \ldots, v_{i}\right\rangle$ the linear subspace generated by the vectors $v_{1}, \ldots, v_{i} \in \mathbb{R}^{d}$; with $\pi_{p}$ the projection on the first $p$ components; with $\lfloor\cdot\rfloor$ the floor function; with $\operatorname{frac}(\cdot)$ the fractional part. $\diamond$

Definition 1. The uniform partition of rate $\delta$ of $\mathbb{R}$ is

$$
\mathscr{P}=\left\{\mathscr{P}_{i}\right\}_{i \in \mathbb{Z}}=\left\{\left[i \delta,(i+1) \delta[\}_{i \in \mathbb{Z}} \cdot \diamond\right.\right.
$$

In this paper we study discrete-time, time-invariant, outputquantized Multi-Input Single Output (MISO) linear systems of the form

$$
\left\{\begin{array}{l}
x(k+1)=A x(k)+B u(k) \\
y(k)=q_{\mathscr{P}}(C x(k))
\end{array}\right.
$$

where $x(k) \in \mathbb{R}^{d}$ is the state, $y(k) \in \mathbb{Z}$ is the output, $u(k) \in \mathscr{U} \subset$ $\mathbb{R}^{m}$ is the input, and $A, B, C$ are matrices of suitable dimensions. The map $q_{\mathscr{P}}: \mathbb{R} \rightarrow \mathbb{Z}$ is induced by the uniform partition $\mathscr{P}=\left\{\mathscr{P}_{i}\right\}_{i \in \mathbb{Z}}$ of $\mathbb{R}$ of rate $\delta$ through $q_{\mathscr{P}}:\left(x \in \mathscr{P}_{i}\right) \mapsto i$ and will be referred to as the output quantizer. We assume that $\mathscr{U}$ is a finite set of cardinality $n$.

Remark 1. Suitably changing the bases, we can suppose the system (1) of the form

$$
\left\{\begin{array}{l}
x(k+1)=A x(k)+B u(k) \\
y(k)=\left\lfloor\pi_{1} x(k)\right\rfloor \diamond
\end{array}\right.
$$

Together with the system (2), we define two "auxiliary" systems, the doubled system and the difference system: the former is useful to have a different point of view on left invertibility, while the latter leads to the definition of left D-invertibility.

Definition 2. The doubled system is

$$
X(k+1)=\left(\begin{array}{cc}
A & 0 \\
0 & A
\end{array}\right) X(k)+\left(\begin{array}{cc}
B & 0 \\
0 & B
\end{array}\right) U(k),
$$

where $X(k)=\left(\begin{array}{c}x(k) \\ x^{\prime}(k)\end{array}\right) \in \mathbb{R}^{2 d}, U(k)=\left(\begin{array}{c}u(k) \\ u^{\prime}(k)\end{array}\right) \in \mathscr{U} \times \mathscr{U} . \diamond$

Definition 3. The difference system is

$$
z(k+1)=A z(k)+B v(k)
$$

where $z(k) \in \mathbb{R}^{d}, v(k) \in \mathscr{U}-\mathscr{U}=\left\{u-u^{\prime}: u, u^{\prime} \in \mathscr{U}\right\} . \diamond$

Roughly speaking, the definition of left invertibility requires that any difference in the input must result in a difference in the following output symbols, at most in a time equal to the invertibility time.

Definition 4. A pair of input strings $\{u(i)\}_{i \in \mathbb{N}},\left\{u^{\prime}(i)\right\}_{i \in \mathbb{N}}$ is uniformly distinguishable in $k$ steps if there exists $l \in \mathbb{N}$ such that $\forall x(0), x^{\prime}(0) \in \mathbb{R}^{d}$ and $\forall m>l$ the following holds for the correspondent trajectories:

$$
\begin{gathered}
u(m) \neq u^{\prime}(m) \Rightarrow \\
{[y(m+1), \ldots, y(m+k)] \neq\left[y^{\prime}(m+1), \ldots, y^{\prime}(m+k)\right],}
\end{gathered}
$$

(outputs $y(i)$ are referred to the system (2) with initial condition $x(0)$ and inputs $u(i)$, while outputs $y^{\prime}(i)$ are referred to the system (2) with initial condition $x^{\prime}(0)$ and inputs $\left.u^{\prime}(i)\right) . \diamond$

Definition 5. The system (2) is uniformly left invertible (ULI) in $k$ steps if every pair of distinct input strings is uniformly distinguishable in $k$ steps after a time $l$, with $k, l$ constant. $\diamond$

The motivation of such a definition is that, for a ULI system, it is possible to recover the input string until instant $m$ observing the output string until instant $m+k$. We now introduce left D-invertibility, and its "finite resolution" version, left $D_{\varepsilon^{-}}$ invertibility: they both are based on the dynamics of the difference system. The difference system represents at any instant the difference between the two states $z(k)=x(k)-x^{\prime}(k)$ when the input symbols $u(k)-u^{\prime}(k)=v(k)$ are performed. So we are interested in understanding the conditions under which

$$
z(k) \notin]-1,1\left[\times\left\langle e_{2}, \ldots, e_{d}\right\rangle\right.
$$

for some $k$. This implies that in system (2) $y(k) \neq y^{\prime}(k)$ (the converse is not true).

Definition 6. A pair of input strings $\{u(i)\}_{i \in \mathbb{N}},\left\{u^{\prime}(i)\right\}_{i \in \mathbb{N}}$ is uniformly D-distinguishable in $k$ steps if there exists $l \in \mathbb{N}$ such that $\forall x(0), x^{\prime}(0) \in \mathbb{R}^{d}$ and $\forall m>l$ the following holds:

$v(m) \neq 0 \Rightarrow[z(m+1), \ldots, z(m+k)] \notin \underbrace{]-1,1[\times \ldots \times]-1,1[}_{k \text { times }}$,

where the $z(i)$ 's are generated by the difference system with initial condition $z(0)=x(0)-x^{\prime}(0)$ and inputs $v(i)=u(i)-$ $u^{\prime}(i) . \diamond$

Definition 7. A system of type (2) is uniformly left D-invertible (ULDI) in $k$ steps if every pair of distinct input strings is uniformly D-distinguishable in $k$ steps after a time $l$, with $k, l$ constant. $\diamond$

Definition 8. Fix $\varepsilon \geq 0$. A pair of input strings is uniformly $D_{\varepsilon}$-distinguishable in $k$ steps if the conditions of Definition 6 are satisfied with ] $-1+\varepsilon, 1-\varepsilon$ [ instead of ] $-1,1[$. A system of type (2) is uniformly left $D_{\varepsilon}$-invertible $\left(U L D_{\varepsilon} I\right)$ in $k$ steps if every pair of distinct input strings is uniformly $D_{\varepsilon^{-}}$ distinguishable in $k$ steps after a time $l$, with $k, l$ constant. $\diamond$

Definition 9. Define the quantization-diagonal set $Q$ relative to the system (2) to be the set of pairs of states $\left(x, x^{\prime}\right) \in \mathbb{R}^{2 d}$ such that $\left\lfloor\pi_{1} x\right\rfloor=\left\lfloor\pi_{1} x^{\prime}\right\rfloor$ i.e. $Q$ contains all pairs of states that are in the same element of the partition $\mathscr{P} . \diamond$

Definition 10. Define $Q_{\varepsilon}$, for $\varepsilon \geq 0$, to be the set of pairs of states $\left(x, x^{\prime}\right) \in \mathbb{R}^{2 d}$ such that $x^{\prime}-x \in(]-1+\varepsilon, 1-\varepsilon[) \times$ $\left\langle e_{2}, \ldots, e_{d}\right\rangle . \diamond$

To address invertibility, we are interested in the dynamics of the doubled system relative to the quantization-diagonal set. If there exists sequences $\{u(k)\},\left\{u^{\prime}(k)\right\}$, and an initial state in the quantization-diagonal set such that the corresponding trajectory of (3) remains in $Q$, then the two strings of inputs generate the same output for the system (2). So conditions ensuring that the state is outside $Q$ for some $k$ will be sought to guarantee left 
invertibility. In the same way we are interested in the dynamics of the difference system relative to the set $Q_{\varepsilon}$. For a graphic illustration of $Q$ and $Q_{\varepsilon}$ see also fig. 1.

Remark 2. Note that Uniform Left $D_{\varepsilon}$-Invertibility (resp. Uniform Left D-Invertibility) means that there do not exist trajectories of arbitrary length in $Q_{\varepsilon}$ (resp. $Q_{0}$ ). Therefore ULDI implies ULI.

The problem under study is to state conditions for the equivalence between ULDI and ULI of the system (1). This equivalence is important since ULDI is algorithmically checkable:

Theorem 1. Dubbini [2010] Consider the system (2) and suppose that: (i) if $\lambda$ is an eigenvalue of the matrix $A$, then $|\lambda| \neq$ 1; (ii) $A$ does not have an invariant subspace in $\left\langle e_{2}, \ldots, e_{d}\right\rangle$. Then, there exists an algorithmic procedure to check left Dinvertibility and find out the invertibility time. $\diamond$

The techniques used in this paper are based on the following Lemma 1: it involves algebraic conditions based on fractional parts to guarantee that the presence of a trajectory in $Q_{\varepsilon}$ implies the existence of a trajectory in $Q$.

Definition 11. If $X \in Q_{\varepsilon}$, define $d(X)$ to be the distance, measured along the line (the $t_{i}$ 's and $\left.s \in\right]-1+\varepsilon, 1-\varepsilon[$ are to be considered fixed)

$$
\left\{\left[\tau, t_{2}, \ldots, t_{d}, \tau+s, t_{d+2} \ldots, t_{2 d}\right] \in \mathbb{R}^{2 d}: \tau \in \mathbb{R}\right\}
$$

from the set $\left\{X \in \mathbb{R}^{2 d}: X_{1}=X_{d+1}=0\right\} . \diamond$

Lemma 1. Suppose that there exists a trajectory of the doubled system $\{X(j)\}_{j=0}^{J} \subset Q_{\varepsilon}$, such that

$$
\operatorname{frac}\left(\frac{d(X(j))}{\sqrt{2}}\right)<\varepsilon,
$$

for every $j=1, \ldots, J$. Then the system (2) is not ULI in $J$ steps.

Proof: First observe that $\operatorname{frac}\left(\frac{d(X(j))}{\sqrt{2}}\right)=0$ if and only if $\left[X_{1}(j), X_{d+1}(j)\right]$ belongs to some translation of $[0,1] \times[0,1]$ along the diagonal of $\mathbb{R}^{2}$ that is entirely included in $Q$, i.e. a translation that takes $[0,1] \times[0,1]$ to the "bottom-left boundary" of a square of $Q$. So, if $X(j) \in Q_{\varepsilon}$ and $\operatorname{frac}\left(\frac{d(X(j))}{\sqrt{2}}\right)<\varepsilon$, then $X(j) \in Q$. Therefore, if the relations (5) are satisfied for $j=1, \ldots, J$, then there exists a proper trajectory of length $J$ included in $Q$, and the system is not ULI in $J$ steps. $\diamond$

Keeping in mind Lemma 1 , we define a quantity $\varepsilon(A)$ depending only on the dynamic matrix $A$ in such a way that, if $\varepsilon(A)<\varepsilon$, then $U L I$ implies $U L D_{\varepsilon} I$.

Definition 12. Given a MISO system of type (2), define

$$
\varepsilon(A)=\sup _{\zeta_{j} \in \mathbb{R}^{d}} \sup _{J \in \mathbb{N}} \inf _{x \in \mathbb{R}^{d}} \max _{j \in[1, J]}\left[\operatorname{frac}\left(\pi_{1}\left(A^{j} x+\zeta_{j}\right)\right)\right] \cdot \diamond
$$

Let us explain the meaning of $\varepsilon(A)$. Suppose we are given any trajectory $\{X(j)\}_{j \in \mathbb{N}}$, included in $Q_{\varepsilon}$, of the doubled system (3). This trajectory has the form

$$
X(j)=\left(\begin{array}{c}
x(j) \\
x^{\prime}(j)
\end{array}\right)=\left(\begin{array}{c}
A^{j} x(0)+A^{j-1} u(1)+\ldots+u(j) \\
A^{j} x^{\prime}(0)+A^{j-1} u^{\prime}(1)+\ldots+u^{\prime}(j)
\end{array}\right) .
$$

We then add the same generic parameter $x \in \mathbb{R}^{d}$ to the first and the second $d$ components of the initial condition (which does not affect the membership to $Q_{\varepsilon}$ ), and consider constant every quantity which does not depend on the parameter:

$$
X(j)=\left(\begin{array}{c}
A^{j}(x+x(0))+\ldots+u(j) \\
A^{j}\left(x+x^{\prime}(0)\right)+\ldots+u^{\prime}(j)
\end{array}\right)=\left(\begin{array}{c}
A^{j} x+\zeta_{j} \\
A^{j} x+\zeta_{j}^{\prime}
\end{array}\right)
$$

So, with a look at Lemma 1 , we can investigate ULI looking at fractional parts of $\pi_{1}\left(A^{j} x+\zeta_{j}\right)$, for $j=1, \ldots, J$, for every $J \in \mathbb{N}$. Taking the sup on $\zeta_{l}$ in the Definition 12 we are not considering the effect of the inputs, so $\varepsilon(A)$ is the smallest $\varepsilon$ such that for every $J \in \mathbb{N}$ there exists a trajectory of the system (2) such that $\operatorname{frac}\left(\pi_{1}\left(A^{j} x+\zeta_{j}\right)\right)<\varepsilon \Rightarrow \operatorname{frac}\left(\frac{d(X(j))}{\sqrt{2}}\right)<\varepsilon \quad j=1 \ldots, J$. Lemma 1 indicates that $\varepsilon(A)$ is the right quantity to put in relation ULDI with ULI:

Proposition 1. Consider the system (2). Then ULI implies $U L D_{\varepsilon(A)} I$.

Proof: Suppose that the system (2) is not $U L D_{\varepsilon(A)} I$. We prove that it is not $U L I$. By $U L D_{\varepsilon(A)} I$ there exists an arbitrary long trajectory of the doubled system in $Q_{\varepsilon(A)}$. Following the reasoning above, we can consider this trajectory of the form (7), and it is possible to choose the initial state such that $\operatorname{frac}\left(\pi_{1} X(j)\right)<$ $\varepsilon(A)$ by the definition of $\varepsilon(A)$ itself, for every $j=1, \ldots, J$. Therefore we can apply Lemma 1 to conclude that the system is not ULI. $\diamond$

\subsection{Mathematical background}

Definition 13. The numbers $\vartheta_{1}, \ldots, \vartheta_{M} \in \mathbb{R}$ are linearly independent over $\mathbb{Z}$ if for every $k_{1}, \ldots, k_{M} \in \mathbb{Z}$ the following holds:

$$
k_{1} \vartheta_{1}+\ldots,+k_{M} \vartheta_{M}=0 \Rightarrow k_{1}=\ldots=k_{M}=0 . \diamond
$$

Theorem 2. (Kronecker). Hardy [1979] If $\alpha_{1}, \ldots, \alpha_{M}, 1 \in \mathbb{R}$ are linearly independent over $\mathbb{Z}$, then, for every $\vartheta_{1}, \ldots, \vartheta_{M} \in \mathbb{R}$ the set of points $\left\{\left[\operatorname{frac}\left(l \alpha_{1}+\vartheta_{1}\right), \ldots, \operatorname{frac}\left(l \alpha_{M}+\vartheta_{M}\right)\right]: l \in \mathbb{R}\right\}$ is dense in the unit cube of $\mathbb{R}^{M} . \diamond$

Definition 14. A number $\rho \in \mathbb{C}$ is called algebraic if there exists a polynomial with integer coefficients $R(x) \in \mathbb{Z}[x]$ such that $R(\rho)=0$. In this case there exists a unique monic polynomial $R(x) \in \mathbb{Z}[x]$ with minimal degree $q . R(x)$ is called the minimal polynomial of $\rho$ and $q$ its degree. A number $\rho \in \mathbb{C}$ is called trascendental if it is not algebraic. $\diamond$

Note that $1, \rho, \rho^{2}, \ldots, \rho^{M}$ are linearly independent if and only if the degree of $\rho$ is at least $M+1$.

Definition 15. If $R(x)$ is the polynomial $R(x)=\sum_{i=0}^{q} r_{i} x^{i}=r_{q}$. $\prod_{j=1}^{q}\left(x-\rho_{j}\right)$, where the $\rho_{j}$ 's are the roots of the polynomial, its Mahler measure is defined as

$$
\mathfrak{M}(R)=\left|r_{q}\right| \cdot \prod_{j=1}^{q} \max \left\{1,\left|\rho_{j}\right|\right\} .
$$

The Mahler measure of an algebraic number is defined to be the Mahler measure of its minimal polynomial. $\diamond$

The Mahler measure of an algebraic number is a measure of its "algebraic complexity", as it is related to the size of the coefficients of the minimal polynomial, like we show in eq. (8). Definition 16. The $i-t h$ symmetric polynomial in $q$ variables is

$$
\sigma_{i}\left(x_{1}, \ldots, x_{q}\right)=\sum_{1 \leq j_{1} \leq \ldots \leq j_{q}} x_{j_{1}} \cdot \ldots \cdot x_{j_{q}} \cdot \diamond
$$

Since $r_{i}$, the $i$-th coefficient of a minimal polynomial, is equal to $r_{q}$ multiplied by the $i$-th symmetric polynomial of the $\rho_{j}$, which is made of precisely $\left(\begin{array}{c}q \\ i\end{array}\right)$ monomials in the $\rho_{i}$ where each $\rho_{i}$ appears with degree at most 1 , we have that $r_{i}$ is sum of $\left(\begin{array}{l}q \\ i\end{array}\right)$ terms each $\leq \mathfrak{M}(A)$ in absolute value, and consequently 
$\left|r_{i}\right| \leq\left(\begin{array}{c}q \\ i\end{array}\right) \cdot \mathfrak{M}(R), \quad$ for $0 \leq i \leq q$. Then we immediately obtain

$$
\max _{0 \leq i \leq q}\left|r_{i}\right| \leq\left(\begin{array}{c}
q \\
{[q / 2]}
\end{array}\right) \cdot \mathfrak{M}(R)
$$

\section{UNIDIMESIONAL SYSTEMS}

Unidimensional systems assume the following form, from (2):

$$
\left\{\begin{array}{l}
x(k+1)=a x(k)+u(k) \\
y(k)=\lfloor x(k)\rfloor
\end{array}\right.
$$

where $x(k) \in \mathbb{R}, y(k) \in \mathbb{Z}, u(k) \in \mathscr{U} \subset \mathbb{R}$, and $a \in \mathbb{R}$.

We now state results about estimates for $\varepsilon(a)$, which will be fundamental in the proofs of finiteness theorems asserting the equivalence between $U L I$ and $U L D I$.

Theorem 3. Dubbini [2009]

- If $a$ is trascendental, then $\varepsilon(a)=0$;

- If $a=\frac{p}{q} \in \mathbb{Q}$, with $\operatorname{gcd}(p, q)=1$, then $\varepsilon(a) \leq \min \left\{\frac{1}{|p|}, \frac{1}{|q|}\right\}$;

- If $a \in \mathbb{R}$ is algebraic of degree $K$ with minimal polynomial $P_{a}(x)$, then

$$
\varepsilon(a) \leq \min \left\{\frac{1}{\mathfrak{M}\left(P_{a}(x / 2)\right)}, \frac{1}{\mathfrak{M}\left(2^{-K} P_{a}(2 x)\right)}\right\} \diamond
$$

Theorem 4. Consider the unidimensional system system (9).

- If $a$ is trascendental, $U L I \Leftrightarrow U L D_{\varepsilon} I$ for every $\varepsilon \geq 0$;

- If $a=\frac{p}{q} \in \mathbb{Q}$, with $\operatorname{gcd}(p, q)=1$, then $U L I$ implies $U L D_{\varepsilon} I$ for every $\varepsilon \geq \min \left\{\frac{1}{|p|}, \frac{1}{|q|}\right\}$;

- If $a$ is algebraic of degree $K$ with minimal polynomial $P_{a}(x)$, then $U L I$ implies $U L D_{\varepsilon} I$ for every

$$
\begin{aligned}
& \varepsilon \geq \operatorname{const}(K) \cdot \min \left\{\frac{1}{\left|\alpha_{i}\right|}: i=1, \ldots, K\right\}, \\
& \text { where } \operatorname{const}(K) \leq \min _{i=1, \ldots, K}\left\{\frac{\left(\begin{array}{c}
K \\
i
\end{array}\right) 2^{\min \{i, K-i\}}}{\left|\alpha_{i}\right|}\right\} .
\end{aligned}
$$

Proof: As to the trascendental and rational case, the thesis of the Theorem follows immediately from Theorem 3, and Proposition 1. In the algebraic statement, first note that in the Theorem 3 the two terms $\mathfrak{M}\left(P_{a}(x / 2)\right)$ and $2^{-K} \mathfrak{M}\left(P_{a}(2 x)\right)$ are the Mahler measures of respectively the polynomial $2^{-K} \alpha_{K} x^{K}+\cdots+$ $2^{-1} \alpha_{1} x+\alpha_{0}$, and the polynomial $\alpha_{K} x^{K}+\cdots+2^{-K+1} \alpha_{1} x+$ $2^{-K} \alpha_{0}$. Moreover by (8) it holds

$$
\begin{gathered}
2^{-i}\left|\alpha_{i}\right| \leq\left(\begin{array}{c}
K \\
i
\end{array}\right) \mathfrak{M}\left(P_{a}(x / 2)\right), \\
2^{-K+i}\left|\alpha_{i}\right| \leq\left(\begin{array}{c}
K \\
i
\end{array}\right) \mathfrak{M}\left(P_{a}(2 x)\right) .
\end{gathered}
$$

The Theorem follows now immediately from Proposition $1 . \diamond$ We now state the main result about unidimensional system, which allows to check uniform left invertibility with an algorithmic procedure (i.e. checking $U L D I$ ), except for a finite number of cases.

Theorem 5. (Finiteness unidimensional Theorem). Consider the unidimensional system (9), and fix the input set $\mathscr{U} \subset \mathbb{R}$. Then, for every $\varepsilon>0$, the set of $a \in \mathbb{R}$ of degree at most $K$ (for any $K \in \mathbb{N}$ ) for which $U L D_{\varepsilon} I$ is not equivalent to ULI is finite.

Proof: Fix an $\bar{\varepsilon}>0$. Consider the periodic trajectory of order 2 of the difference system given by $\left\{\begin{array}{l}a x_{1}+v=x_{2} \\ a x_{2}-v=x_{1}\end{array}\right.$. This system of equation has the solution $x_{1}=\frac{-v}{a+1}, x_{2}=\frac{v}{a+1}$. If

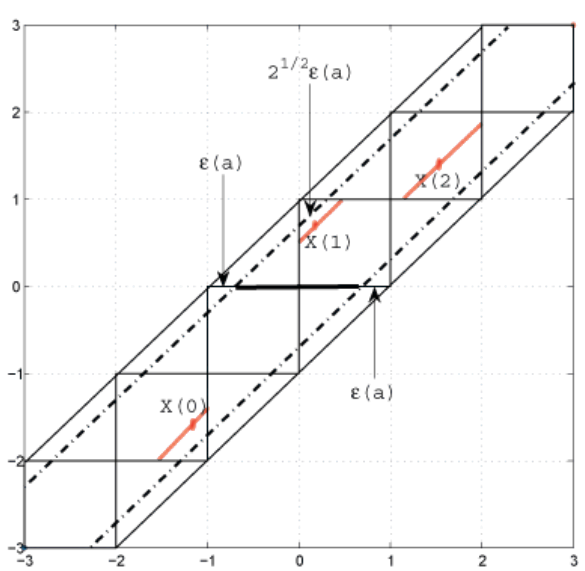

Figure 1 . The set $Q$ (formed by the squares) and the set $Q_{\varepsilon}$ (the dashed "strip") are shown in the case of the unidimensional system (9): Proposition 1 means that a trajectory inside $Q_{\varepsilon(a)}=\{[-1+\varepsilon(a), 1-\varepsilon(a)]+$ $(t, t): t \in \mathbb{R}\}$ imples the existence of a trajectory inside $Q$.

$$
\min _{0 \neq v \in \mathscr{V}}|v|<(a+1)(1-\bar{\varepsilon})
$$

the periodic trajectory $\left(x_{1}, x_{2}, x_{1}, x_{2}, x_{1}, \ldots\right)$ lies in $]-1+\bar{\varepsilon}, 1-$ $\bar{\varepsilon}$ [ and the system is not $U L D_{\bar{\varepsilon}} I$. Theorem 3 implies that (since the degree $K$ is fixed) all but a finite number of $a \in \mathbb{R}$ satisfy

$$
\varepsilon(a)<\bar{\varepsilon}
$$

- Suppose that the system (9) is not $U L D_{\bar{\varepsilon}} I$. Then it is not $U L D_{\varepsilon(a)} I$ every time that (11) holds, and it holds for all but a finite number of $a$ 's. By Proposition 1 the system is then not $U L I$. We have thus proved that $U L I \Rightarrow U L D_{\bar{\varepsilon}} I$ except for a finite number of $a$ 's.

- Suppose now that for a particular $a \in \mathbb{R}$ the system (9) is $U L I$ but not $U L D_{\bar{\varepsilon}} I$. Following the the derivation of (10), it must be

$$
(a+1)(1-\varepsilon(a))<\min _{0 \neq v \in \mathscr{V}}|v|<(a+1)(1-\bar{\varepsilon}) .
$$

Theorem 3 again implies that there are only a finite number of $a$ such that (12) holds, so there are only a finite number of $a$ 's such that the system (9) is $U L I$ but not $U L D_{\bar{\varepsilon}} I$. We have thus proved that $U L D_{\bar{\varepsilon}} I \Rightarrow U L I$ except for a finite number of $a$ 's. $\diamond$

\section{MISO SYSTEMS}

Suppose that $A$ has an eigenvalue $\lambda \in \mathbb{C}$ relative to an eigenvector $w$. Then also its complex conjugate $\bar{\lambda}$ is an eigenvalue, relative to the eigenvector $\bar{w}: A \bar{w}=\bar{\lambda} \bar{w}$. Moreover, denoting with $\operatorname{Re}(\cdot)$ and $\operatorname{Im}(\cdot)$ the real part and the imaginary part of a complex number (or a vector, componentwise) it holds

$$
\begin{gathered}
w_{R e}=\frac{w+\bar{w}}{2}=\operatorname{Re}(w), \quad w_{\operatorname{Im}}=-i \frac{w-\bar{w}}{2}=\operatorname{Im}(w), \\
\pi_{1}\left(A^{k} w_{R e}\right)=\operatorname{Re}\left(\lambda^{k}\right), \quad \pi_{1}\left(A^{k} w_{\operatorname{Im}}\right)=\operatorname{Im}\left(\lambda^{k}\right) .
\end{gathered}
$$

The following Theorem provides a sufficient condition for the equivalence of ULDI and ULI of a MISO system, only on the base of the eigenvalues of the matrix $A$.

Theorem 6. Consider a MISO system of type (2). Define $\varepsilon$ to be the minimum of $\varepsilon(\lambda)$, with $\lambda$ varying among the (also complex) eigenvalues of $A$ whose respective eigenvectors have first component $\neq 0$. If the system is not $U L D_{\varepsilon} I$, then it is not ULI. 
Proof: Suppse that the system is not $U L D_{\varepsilon} I$. Then it has arbitrary long trajectories of the doubled system in $Q_{\varepsilon}$. Note that, if $\{X(k)\}_{k \in \mathbb{N}}$ is such a trajectory, then also the trajectory obtained considering the same input sequence and initial condition $X(0)+t\left(\begin{array}{l}w_{R e}+\tau_{\text {Im }} \\ w_{R e}+\tau_{\text {Im }}\end{array}\right)$, instead of $X(0)$ ( $w$ eigenvector of the matrix $A$ ), is included in $Q_{\varepsilon}$. In particular, assuming that $t$ varies in $\mathbb{R}$, the trajectory assumes the form

$$
\begin{gathered}
\left\{\left(\begin{array}{c}
x(0)+t\left(w_{R e}+\tau w_{I m}\right) \\
x^{\prime}(0)+t\left(w_{R e}+\tau w_{I m}\right)
\end{array}\right),\right. \\
,\left(\begin{array}{c}
A x(0)+A t\left(w_{R e}+\tau w_{I m}\right)+u(0) \\
A x^{\prime}(0)+A t\left(w_{R e}+\tau w_{I m}\right)+u^{\prime}(0)
\end{array}\right), \ldots, \\
\vdots \\
\left.\left(\begin{array}{c}
A^{k} x(0)+A^{k} t\left(w_{R e}+\tau w_{I m}\right)+\ldots+u(k-1) \\
A^{k} x^{\prime}(0)+A^{k} t\left(w_{R e}+\tau w_{I m}\right)+\ldots+u^{\prime}(k-1)
\end{array}\right), \ldots\right\} \\
=\left\{\left(\begin{array}{c}
t\left(w_{R e}+\tau w_{I m}\right) \\
t\left(w_{R e}+\tau w_{I m}\right)
\end{array}\right)+\operatorname{const}(0), \ldots\right. \\
\left.\ldots,\left(\begin{array}{c}
A^{k} t\left(w_{R e}+\tau w_{I m}\right) \\
A^{k} t\left(w_{R e}+\tau w_{I m}\right)
\end{array}\right)+\operatorname{const}(k), \ldots\right\} .
\end{gathered}
$$

We are considering constants all the values that are not influenced by $t$. The projection corresponding to component 1 and $d+1$ of this trajectory is simply

$$
\begin{gathered}
=\left\{t\left(\begin{array}{l}
1 \\
1
\end{array}\right)+\operatorname{const}(0), \ldots\right. \\
\left.\ldots, t\left(\begin{array}{l}
\left(\operatorname{Re}\left(\lambda^{k}\right)+\tau \operatorname{Im}\left(\lambda^{k}\right)\right) \\
\operatorname{Re}\left(\lambda^{k}\right)+\tau \operatorname{Im}\left(\lambda^{k}\right)
\end{array}\right)+\operatorname{const}(k), \ldots\right\} .
\end{gathered}
$$

Now choose $\tau$ to be a trascendental number. If there are integer linear relations among the component of (13), i.e.:

$$
\sum_{i=1}^{k} l_{i}\left[\operatorname{Re}\left(\lambda^{i}\right)+\tau \operatorname{Im}\left(\lambda^{i}\right)=0\right], \quad l_{i} \in \mathbb{Z}
$$

then it must be

$$
\sum_{i=1}^{k} l_{i} \operatorname{Re}\left(\lambda^{i}\right)=0, \quad \sum_{i=1}^{k} l_{i} \operatorname{Im}\left(\lambda^{i}\right)=0,
$$

because of the choice of a trascendental $\tau$. Moreover equations (14) hold if and only if the complex number $\lambda$ satisfies the same integer linear relation:

$$
\sum_{i=1}^{k} l_{i} \lambda^{i}=0
$$

The estimates given in Theorem 3 depend only on the coefficients of the minimal polynomial of an algebraic number, and linear relations (14) are the same of (15). This implies that $\varepsilon\left(\operatorname{Re}\left(\lambda^{k}\right)+\tau \operatorname{Im}\left(\lambda^{k}\right)\right)=\varepsilon(\lambda)$, and that estimates given in the Theorem 3 are valid considering the minimal polynomial of $\lambda$. Therefore there exists a $t \in \mathbb{R}$ such that the trajectory $\{X(k)\}_{k \in \mathbb{N}}$ satisfies Lemma 1 with $\varepsilon=\varepsilon(\lambda)$. This proves the Theorem, since we can do the same for every eigenvalue $\lambda$, choosing the one with minimum $\varepsilon(\lambda) . \diamond$

Corollary 1. If the matrix $A$ has a trascendental eigenvalue with respective eigenvector having the first component $\neq 0$, the system is ULDI if and only if it is ULI. $\diamond$

Corollary 2. If the matrix $A$ has an algebraic eigenvalue $\lambda$ with respective eigenvector having the first component $\neq 0$, and the system is not $U L D_{\varepsilon(\lambda)} I$, then it is not ULI. $\diamond$

\section{AN EXAMPLE}

Example 1. Consider the system

$$
\left\{\begin{array}{l}
x(k+1)=\left(\begin{array}{cc}
1 & -1 \\
3 & 2
\end{array}\right) x(k)+\left(\begin{array}{c}
1 \\
1
\end{array}\right) u(k) \\
y(k)=\left\lfloor\pi_{1} x(k)\right\rfloor ; \mathscr{U}=\left\{-\frac{3}{5}, \frac{3}{5}\right\},
\end{array}\right.
$$

The (complex) eigenvalues of the matrix $A$ are $\frac{3 \pm \sqrt{-11}}{2}$, given by the characteristic, and minimal, polynomial $\lambda^{2}-3 \lambda+5=0$. Moreover, both eigenvectors have non null first component. Therefore we can apply Theorem 3 to conclude that

$$
\varepsilon(A) \leq \min _{i=0,1,2}\left\{\frac{2 \cdot 2^{\left\lfloor\frac{i}{2}\right\rfloor}}{\left|\alpha_{i}\right|}\right\}=\frac{2}{5},
$$

where $\left|\alpha_{i}\right|$ is the modulus of the $i$-th coefficient of the minimal polynomial of the matrix $A$. We show that the system (16) is not $U L D_{\frac{2}{5}} I$, so that by Proposition 1 the system is not ULI. To check $U L D_{\frac{2}{5}} I$ we applied the following algorithm:

(1) Compute the difference system, and the polytope

$$
P 0=]-1+\frac{2}{5}, 1-\frac{2}{5}\left[\times\left\langle e_{2}\right\rangle ;\right.
$$

(2) Compute the following sequence of polytopes, starting from $\mathrm{P} 0$ and iterating:

$$
P(k+1)=P 0 \cap \bigcup_{v_{i} \in \mathscr{U}-\mathscr{U}} A(P(k))+B v_{i} ;
$$

The polytope $P(k)$ represents the set of possible states of the difference system that are in $]-1+\frac{2}{5}, 1-\frac{2}{5}\left[\times\left\langle e_{2}\right\rangle\right.$ after $k$ steps.

(3) If $P(k)$ is empty for some $k$, the system is $U L D_{\frac{2}{5}} I$ in time $k$, since no state can be in $]-1+\frac{2}{5}, 1+\frac{2}{5}\left[\times\left\langle e_{2}\right\rangle\right.$ after $k$ iterations. If $P(k+1)=P(k)$ for some $k$ then the system is not $U L D_{\frac{2}{5}} I$, since $P(k)$ is an invariant set for the iterations of the difference system relative to (16).

Note that this algorithm halts thanks to Theorem 1. Fig. 2 shows the polytopes $P(k)$ for $k=1,2,3,4$. The system (16) is not $U L D_{\frac{2}{5}} I$, since $P(4)$ is an invariant set for the difference system. Applying Proposition 1 we conclude that the system is not ULI. $\diamond$

\section{CONCLUSIONS}

In this paper we studied left invertibility of quantized MISO linear systems. We proved that in unidimensional systems it is equivalent, except for a finite number of cases, to left Dinvertibility, while for higher dimension MISO systems we proved a sufficient condition for the equivalence between ULDI and ULI, based on the eigenvalues of the system. Left Dinvertibility is easily (and algorithmically) checkable, so our results allow in practice to check left invertibility of outputquantized MISO linear systems with a computable procedure. Algebraic conditions play a central role in the investigation of left invertibility as well in other fields when a quantization is introduced (Bicchi [1992], Chitour [2001]). Future research will include investigation on the equivalence between left invertibility and left D-invertibility in MIMO systems. 


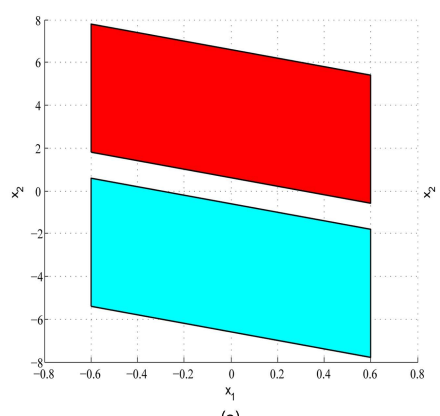

(a)

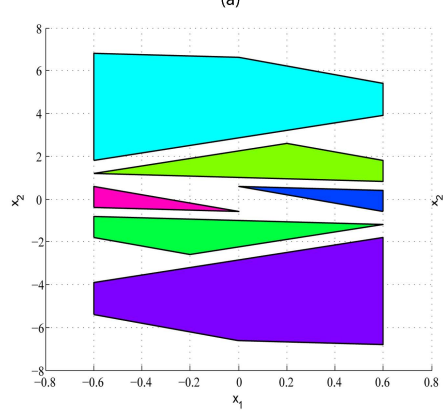

(c)

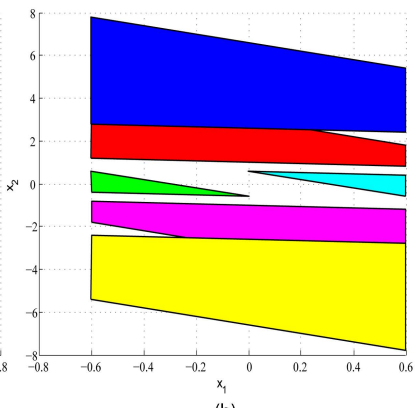

(b)

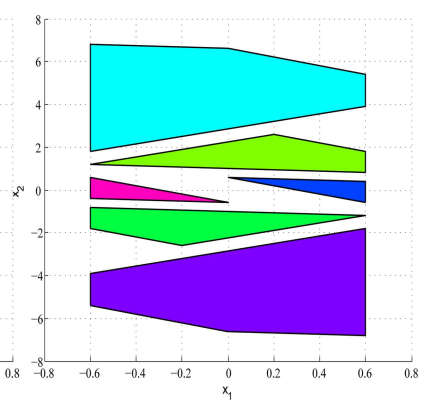

(d)

Figure 2. Polytopes of the difference system relative to (16) representing states inside $]-1+\frac{2}{5}, 1-\frac{2}{5}\left[\times\left\langle e_{2}\right\rangle\right.$ (a) after 1 step, (b) after 2 steps, (c) after 3 steps and (d) after 4 steps.

\section{REFERENCES}

J.E. Bertram. The effect of quantization in sampled feedback systems. AIEE Transactions on Applied Industry, II-77:177$181,1958$.

A. Bicchi, A. Marigo, B. Piccoli. On the reachability of quantized control sytems. IEEE Transactions on Automatic Control, 47(4):546-563, 2002.

R. Brockett, D. Liberzon. Quantized feedback stabilization of linear systems. IEEE Transactions on Automatic Control, 45(7):1279-1289, 2000.

R.W. Brockett, M.D. Mesarovic. The reproducibility of multivariable control systems. Journal of Mathematical Analysis and Applications, 11:548-563, 1965.

R. Carli, F. Fagnani, A. Speranzon, S. Zampieri. Communication constraints in the average consensus problem. Automatica, 44:671-684, 2008.

Y. Chitour, B. Piccoli. Controllability for discrete systems with a finite control set. Mathematics of Control Signal and Systems, 14:173-193, 2001.

R.E. Curry. Estimation and control with quantized measurements. M.I.T. Press, Research Monograph, 1970.

D.F. Delchamps. Stabilizing a linear system with quantized state feedback. IEEE Transactions on Automatic Control, 35(8):916-924, 1990.

N. Dubbini, B. Piccoli, A. Bicchi. Left invertibility of discrete systems with finite inputs and quantized output. International Journal Of Control, 83(4):798-809, 2010.

N. Dubbini, B. Piccoli, A. Bicchi. Left invertibility of discretetime output-quantized systems: the linear case with finite inputs. Mathematics of Control Signal and Systems, submitted, preprint available at http://arxiv.org/abs/1103.4039, 2010.

N. Dubbini, M. Monge. An equivalent of Kroneckers theorem for powers of an algebraic number and structure of linear recurrences of fixed length. Acta Arithmetica, accepted for publication, preprint available at http://arxiv.org/abs/0910.5182, 2010.

N. Dubbini, A. Carluccio, A. Bicchi. Left invertibility of output quantized systems: an applications to cryptography. Proceedings of 49-th IEEE Conference on Decision and Control, 4078-4083, 2010.

A. Edelmayer, J. Bokor, Z. Szabó, F. Szigeti Input reconstruction by means of system inversion: a geometric approach to fault detection and isolation in nonlinear systems. International Journal of Applied Mathematics and Computer Science, 14(2):189-199, 2004.

M. Fliess. A note on the invertibility of nonlinear input-output differential systems. Systems and Control Letters, 8:147$151,1986$.

M. Fu, L. Xie. The Sector Bound Approach to Quantized Feedback Control. IEEE Transactions on Automatic Control, 50(11):1698-1711, 2005.

G.C. Goodwin. Inverse problems with constraints. Proceedings of the 15-th International Federation of Automatic Control, published on CD-ROM, 2002.

G.H. Hardy, E.M. Wright. An introduction to the theory of numbers. Oxford Science Publications, 1979.

R.M. Hirschorn. Invertibility of nonlinear control systems SIAM Journal on Control and Optimization 17(2):289-297, 1979.

R.M. Hirschorn. Invertibility of multivariable nonlinear control systems IEEE Transactions on Automatic Control, AC(24): 855-865, 1979.

R.E. Kalman. Nonlinear aspects of sampled-data control systems. Proceedings of the Symposium on Nonlinear Circuit Theory, VII, Polytechnic Press, 1956.

A.S. Krupadanam, A.M. Annaswamy, R.S. Mangoubi. Multivariable adaptive control design with applications to autonomous helicopters American Institute of Aeronautics and Astronautics Journal of Guidance Control and Dynamics, 25(5):843-851, 2002.

G.N. Nair, F. Fagnani, S. Zampieri, R.J. Evans. Feedback Control Under Data Rate Constraints: An Overview. Proceedings of the IEEE, 95(1):108-137, 2007.

W. Respondek. Right and Left Invertibility of Nonlinear Control Systems. Nonlinear Controllability and Optimal Control, 133-176, 1990.

W. Respondek. Geometry of static and dynamic feedback Lecture notes, 2001.

L.M. Silverman. Inversion of multivariable linear systems. IEEE Transactions on Automatic Control, 14(3):270-276, 1969.

M. Szanier, A. Sideris. Feedback control of quantized constrained systems with applications to neuromorphic controller design. IEEE Transactions on Automatic Control, 39(7):1497-1502, 1994.

F. Szigeti, C.E. Vera, J. Bokor, A. Edelmayer. Inversion based fault detection and isolation. Proceedings of the 40-th IEEE Conference on Decision and Control, 1005-1010, 2001.

A. Tanwani, D. Liberzon. Invertibility of switched nonlinear systems Automatica, 46:1962-1973, 2010.

S.C. Tatikonda, S. Mitter. Control under communication constraints. IEEE Transactions on Automatic Control, 49(7): 1056-1068, 2004.

L. $\mathrm{Vu}, \mathrm{D}$. Liberzon. Invertibility of switched linear systems. Automatica, 44:949-958, 2008. 